\title{
Chaos Analysis and Synchronization Control of Coronary Artery Systems
}

\author{
Cheng-Chi Wang ${ }^{1}$ and Her-Terng Yau ${ }^{2}$ \\ ${ }^{1}$ Graduate Institute of Precision Manufacturing, National Chin-Yi University of Technology, Taichung 41170, Taiwan \\ ${ }^{2}$ Department of Electrical Engineering, National Chin-Yi University of Technology, Taichung 41170, Taiwan
}

Correspondence should be addressed to Her-Terng Yau; pan1012@ms52.hinet.net

Received 15 September 2013; Accepted 27 September 2013

Academic Editor: Carlo Bianca

Copyright (c) 2013 C.-C. Wang and H.-T. Yau. This is an open access article distributed under the Creative Commons Attribution License, which permits unrestricted use, distribution, and reproduction in any medium, provided the original work is properly cited.

\begin{abstract}
The present study uses the differential transformation method to solve the governing equations of the coronary artery system and then analyzes the dynamic behavior of the system by means of phase portraits, power spectra, bifurcation diagrams, and Poincaré maps. Also, a master-slave control system is proposed to suppress the nonlinear chaotic behavior of the coronary artery system. The results show that the dynamic behavior of the coronary artery system is significantly dependent on the magnitude of the vibrational amplitude. Specifically, the motion changes from $T$-periodic to $2 T$-periodic, then from $4 T$-periodic to $8 T$-periodic, and finally to chaotic motion with windows of periodic motion as the vibrational amplitude is increased from 0.3 to 0.6 and from 4.5 to 5.9. In addition, it is shown that the proposed control scheme enables the coronary artery system to be synchronized to any state asymptotically such that the risk of cardiopathy is reduced.
\end{abstract}

\section{Introduction}

Arrhythmias are heart-rhythm problems in which the heart beats too fast, too slow, or irregularly. While many arrhythmias are harmless and cause only relatively minor symptoms such as shortness of breath, dizziness, and sudden weakness, in severe cases arrhythmias can lead to cardiac arrest and even death. There are many reasons for the rhythm change of the coronary artery (CA) system, including thyroid disease, high blood pressure, heart-valve problems, abnormalities of the heart ectopic pacemaker, and irregular dynamic phenomena such as the frequency of the conduction system. The prediagnosis of arrhythmia enables the selection of appropriate antiarrhythmic drugs, thus improving the arrhythmia and reducing the probability of sudden heart problems. Arrhythmia is generally detected by means of electrocardiography (ECG), in which the electrical activity of the heart is measured by means of electrodes attached to the arms, legs, and chest and is then printed out on paper [1-3]. However, while electrocardiograms are essential tools in diagnosing heart disease, the ECG signal is highly nonlinear and is therefore not easily analyzed using traditional methods. Thus, various machine-assisted solutions for diagnosing and monitoring arrhythmia have been proposed in recent decades, including Bayesian methods, heuristic methods, expert systems, Markov models, and artificial neural networks (ANNs) [4].

A typical electrocardiogram includes three basic waveforms, namely, a $\mathrm{P}$ wave, a QRS wave, and a $\mathrm{T}$ wave, where a $\mathrm{P}$ wave represents the wave of depolarization, a QRS complex wave represents ventricular depolarization, and a $\mathrm{T}$ wave represents ventricular repolarization [5]. These waves are the main factors affecting the movement of the heart, and thus their dynamic behaviors must be properly understood if the mechanisms of the HBV system are to become clear [6]. Furthermore, if the cardiac motion can be precisely controlled, the chances of both diagnosing and curing heart disease and abnormalities can be significantly enhanced [7].

This paper applies the differential transformation method to solve the governing equations for the coronary artery (CA) system. The non-linear dynamic behavior of the system is then investigated by means of phase portraits, power spectra, bifurcation diagrams, and Poincaré maps. In addition, a master-slave control system is proposed to suppress the 
TABLE 1: System parameters [9].

\begin{tabular}{lcccc}
\hline Parameter & $b$ & $C$ & $\lambda$ & $\omega$ \\
\hline Value & 0.15 & -1.7 & -0.65 & 1 \\
\hline
\end{tabular}

non-linear chaotic behavior of the CA system. The simulation results show that the proposed controller enables the abnormal slave CA system to be synchronized with the normal master CA system despite the presence of system uncertainties.

\section{Nonlinear Dynamic Analysis}

2.1. Mathematical Modeling. The governing equations of the CA system were originally derived by Guan [8] in 2002 and were subsequently converted to the following form by Gong et al. [9] a few years later:

$$
\begin{gathered}
\dot{x}+b x+c y=0, \\
\dot{y}+(\lambda+b \lambda) x+(\lambda+c \lambda) y-\lambda x^{3}-E \cos \omega t=0,
\end{gathered}
$$

where $x$ is the variation of the blood vessel diameter, $y$ is the change in the blood pressure, $E \cos \omega t$ is the external disturbance factor acting on the blood vessels, and $b, c$, and $\lambda$ are the system parameters.

A nonlinear chaotic behavior of the CA system may result in various cardiovascular problems, including myocardial infarction, angina, and even death. Therefore, the present study proposes a master-slave CA synchronization control system based on the following state equations:

$$
\begin{gathered}
\dot{x}=-b x-c y, \\
\dot{y}=-(\lambda+b \lambda) x-(\lambda+c \lambda) y+\lambda x^{3}+E \cos \omega t,
\end{gathered}
$$

In designing the proposed controller, the CA system parameters are assigned the values shown in Table 1.

2.2. Differential Transformation Method. Applying the differential transformation method [10-12] to (2) with respect to the time domain $t$, the two state equations of the coronary artery system become

$$
\begin{aligned}
\frac{k+1}{H} \bar{X}(k+1)= & -b \bar{X}(k)-c \bar{Y}(k), \\
\frac{k+1}{H} \bar{Y}(k+1)= & -(\lambda+b \lambda) \bar{X}(k)-(\lambda+c \lambda) \bar{Y}(k) \\
& +\lambda \bar{X} \otimes \bar{X} \otimes \bar{X}+E \frac{(\omega H)}{k !} \cos \left(\frac{\pi k}{2}\right),
\end{aligned}
$$

respectively (note that the fundamental properties of the Taylor transformation are provided in the appendix).

In the present study, the dynamic behavior of the CA system is characterized by means of the phase portraits, power spectra, bifurcation diagrams, and Poincaré maps produced using the time-series data for parameters $x$ and $y$ of the CA system. Note that, sin producing the various plots, the time-series data corresponding to the first 1000 revolutions are deliberately excluded in order to ensure that the results relate to steady-state conditions.

\subsection{Numerical Results for Nonlinear Dynamic Behavior}

2.3.1. Phase Portraits and Power Spectra. Figure 1 shows the phase portraits of $X$ and $Y$ for various values of the vibrational amplitude, E. Figures 1(a) and 1(c) show that the orbit is regular at $E=0.1$ and 4.3 but is irregular and nonperiodic at $E=0.3$ and 4.5 as shown in Figures $1(\mathrm{~b})$ and $1(\mathrm{~d})$. Furthermore, at higher values of the vibrational amplitude, that is, $E=5.9$ and 16.3, respectively, the orbit exhibits regular, periodic motion (see Figures $1(\mathrm{e})$ and $1(\mathrm{f})$ ).

Figures 2(a)-2(f) present the power spectra for the variation of the blood vessel diameter and the change in blood pressure of the CA system. It is seen that, for vibrational amplitudes of $E=0.1$ and 4.3, the CA system performs $T$ periodic and $8 T$-periodic motion. However, for vibrational amplitudes of $E=0.3$ and 4.5 , the system exhibits chaotic motion. Finally, for vibrational amplitudes of $E=5.9$ and 16.3 , the system exhibits multiperiodic behavior.

2.3.2. Bifurcation Analysis. Figures 3(a) and 3(b) show the bifurcation diagrams for the variation of the blood vessel diameter and change in blood pressure, respectively. In addition, Figures 4(a)-4(f) present the Poincaré maps of the system trajectory given vibrational amplitudes of $E=0.1,0.3$, $0.6,2.1,3.8,4.3,4.4,4.5,5.9,6.0,6.2$, and 16.3 , respectively. Figures 3(a) and 3(b) show that, at lower values of the vibrational amplitude, that is, $E<0.3$, the variation of the bloodvessel diameter $(X)$ and the change in blood pressure $(Y)$ both exhibit a dynamic periodic response. Figure 4(a) presents the Poincaré map corresponding to $E=0.1$. It is seen that the orbit behaves periodic motion as shown and proven in Figure 1(a) and corresponding to a single point on Poincaré map. As the value of the vibrational amplitude is increased from $E=0.3$ to $E=0.59$, the system performs chaotic motion, as shown in Figure 4(b). At $E=0.6$, the chaotic motion is replaced by $T$-periodic motion. Figure 3 shows that this $T$-periodic motion is maintained for all values of the vibrational amplitude in the range of $0.6 \leq E<2.1$. However, as the vibrational amplitude is increased to $E=3.8$, the $T$-periodic motion loses its stability and is replaced by $2 T$-periodic (subharmonic) motion. As $E$ is further increased over the interval $3.8 \leq E<4.5$, the system exhibits multiperiodic motion comprising both $4 T$ - and $8 T$-periodic motions, as shown in Figures 4(c) and 4(d). However, at $E=4.5$, the multiperiodic motion is replaced by chaotic motion $[13,14]$. For values of the vibrational amplitude in the range of $4.5 \leq E<5.9$, the system performs chaotic motion (see Figure 4(e)). However, as $E$ is increased over the interval $5.9 \leq E \leq 20.0$, the system exhibits multiperiodic motion once again, including $8 T$-periodic, $6 T$-periodic, $3 T$-periodic, and $4 T$-periodic, as shown in Figure 4(f), corresponding to $E=16.3$. 


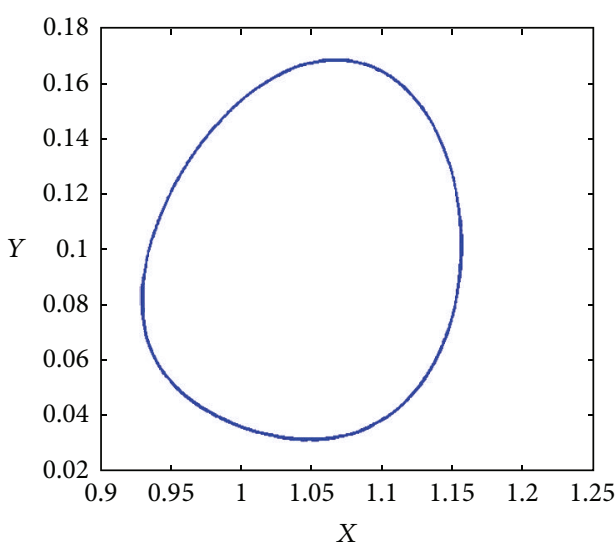

(a)

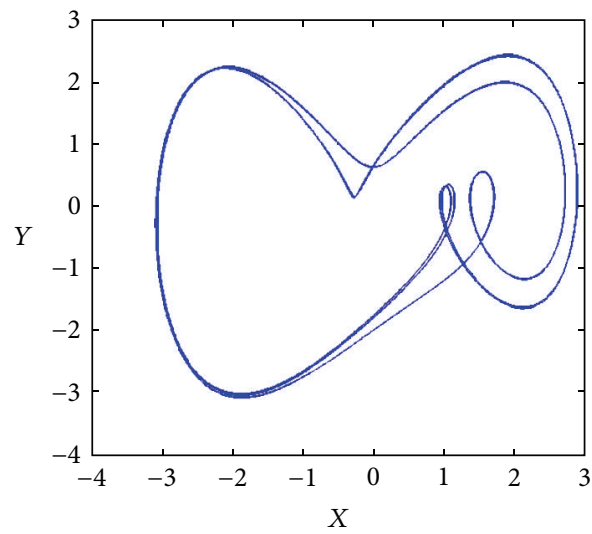

(c)

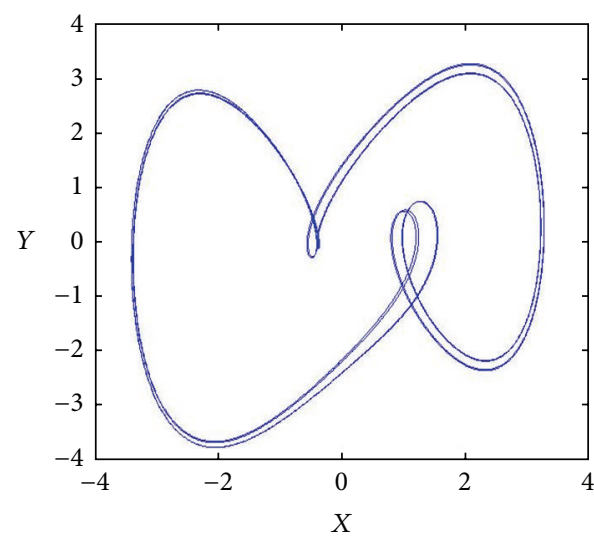

(e)

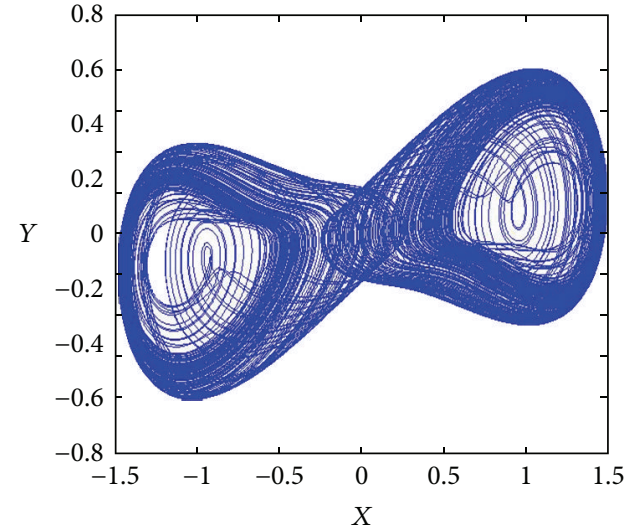

(b)

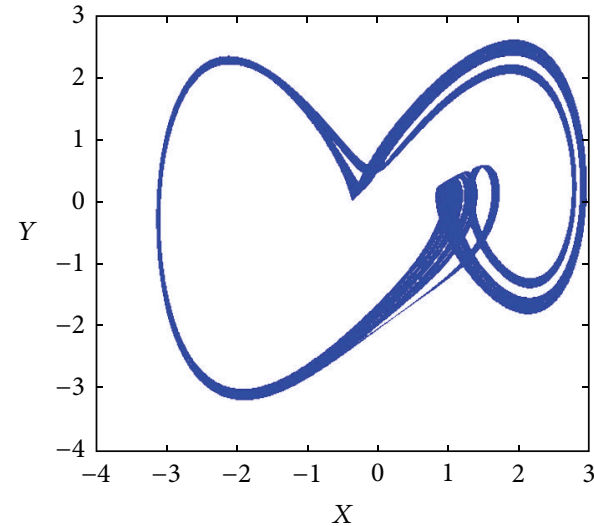

(d)

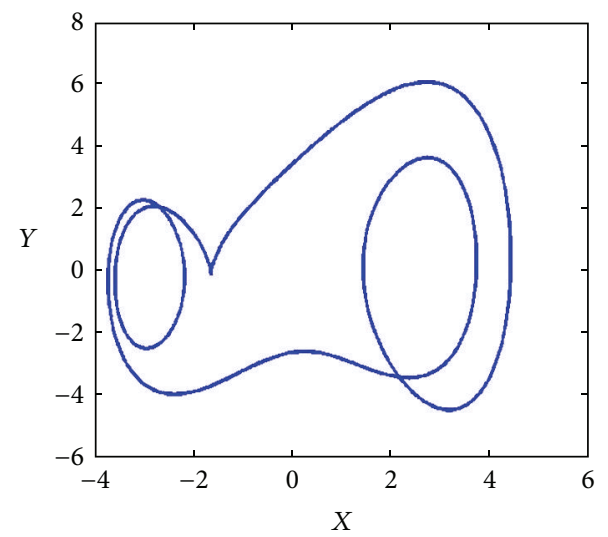

(f)

FIGURE 1: Phase portraits of coronary artery system at $E=$ (a) 0.1 , (b) 0.3 , (c) 4.3 , (d) 4.5 , (e) 5.9 , and (f) 16.3 .

From the above discussions, it is clear that the dynamic response of the CA system depends heavily on the magnitude of the vibrational amplitude. The various motions performed by the system as the vibrational amplitude is increased from $E=0.1$ to $E=20.0$ are summarized in Table 2. In general, the results show that, depending on the value of the vibrational amplitude, the CA system may exhibit periodic behavior, that is, $T$-, $2 T$-, $4 T$-, or $8 T$-periodic motion, or a chaotic response. In addition, it is noted that an explosive bifurcation occurs at a vibrational amplitude of $E=0.3$; the system has a chaotic state with windows of periodic motion.

\section{Chaos Synchronization Control}

From the above analysis, it can be seen that the coronary artery system have very complex behaviour. In this section, it will be studied to design a controller to synchronize the 


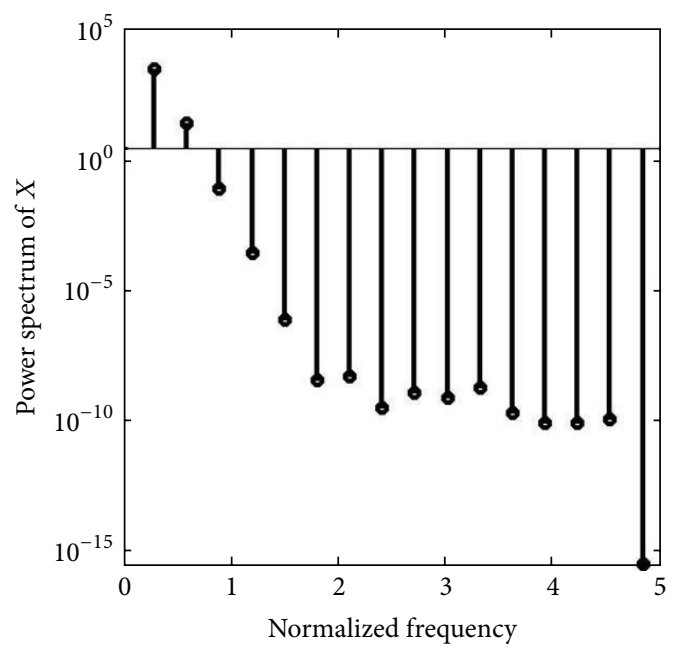

(A)

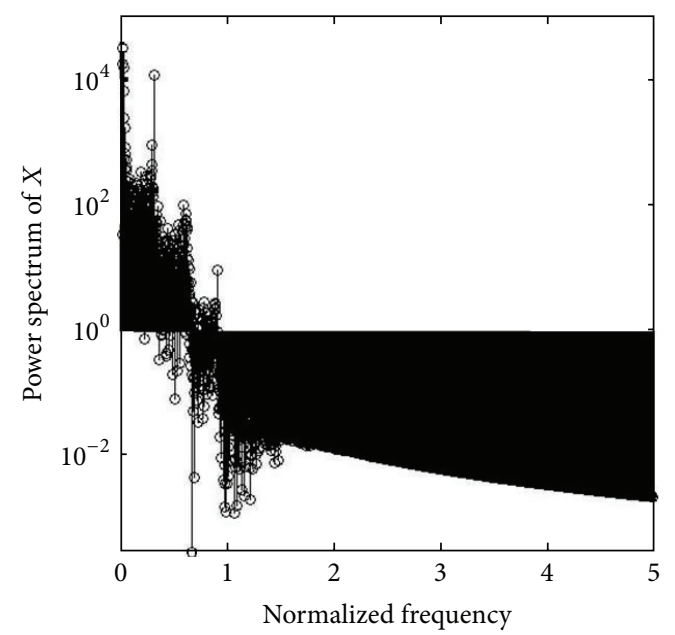

(A)

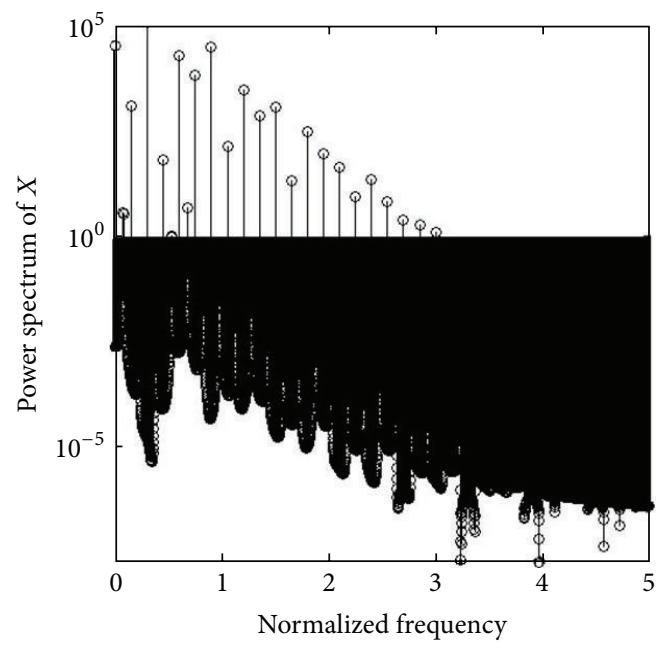

(A)

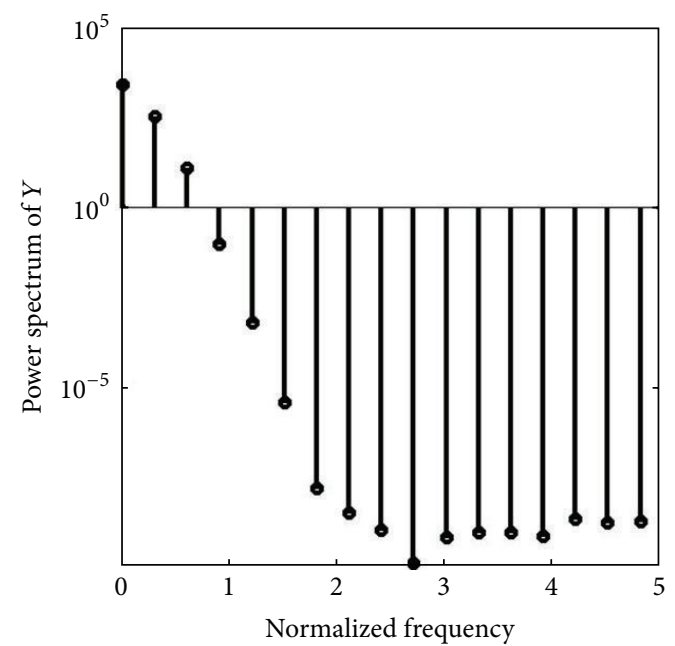

(B)

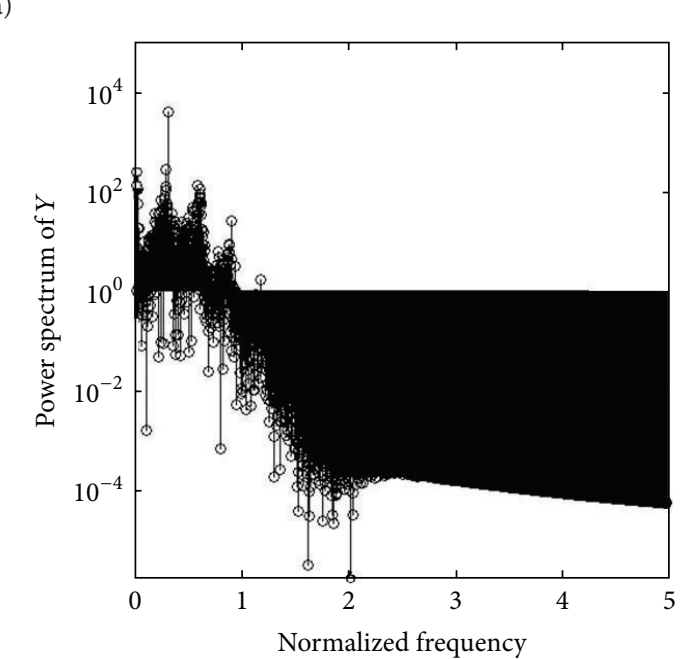

(B)

(b)

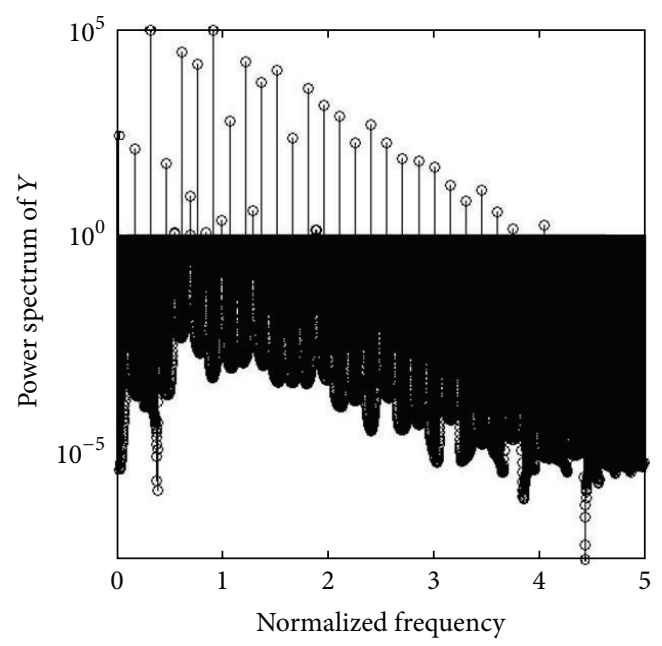

(B)

(c) 


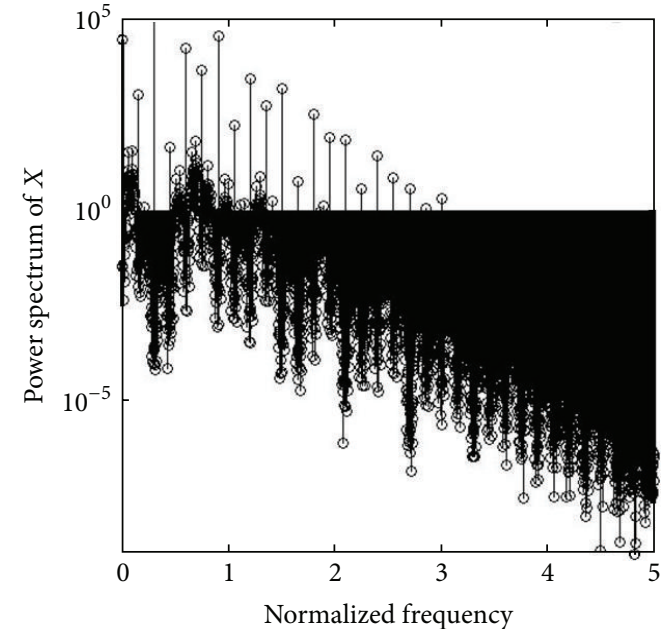

(A)

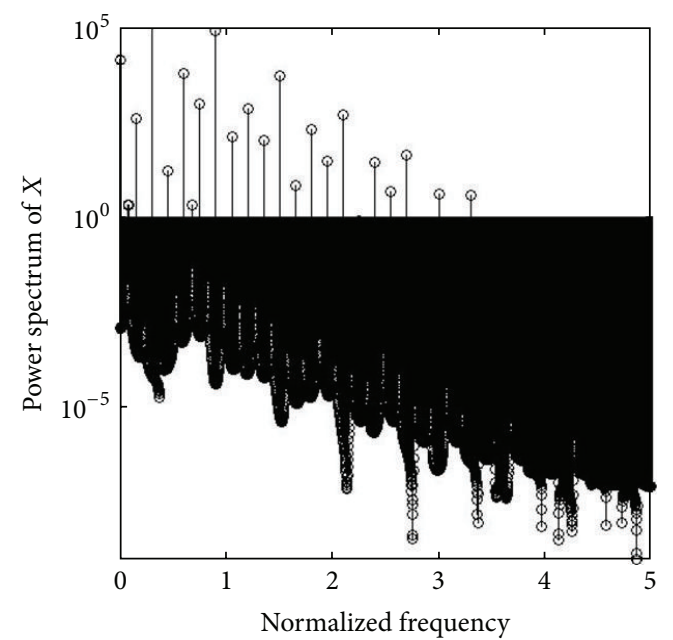

(A)

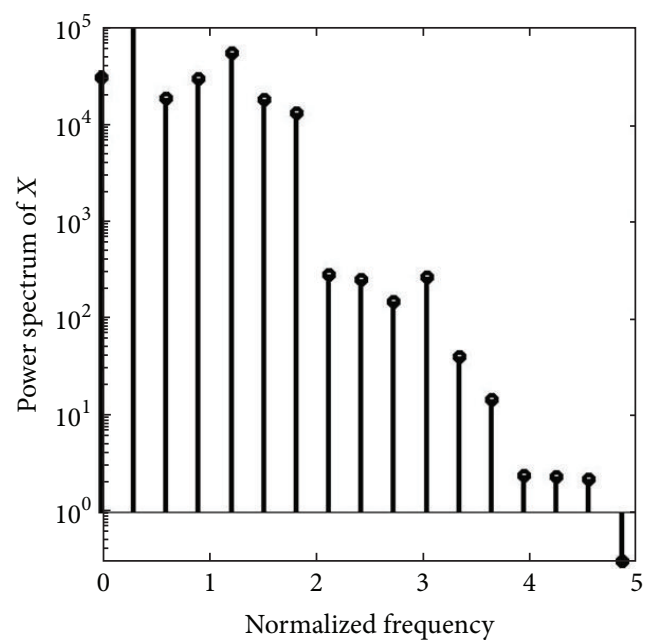

(A)

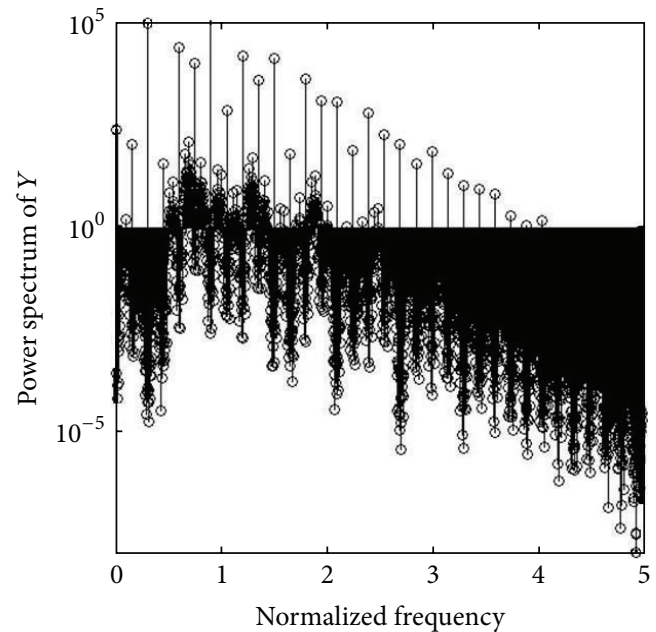

(B)

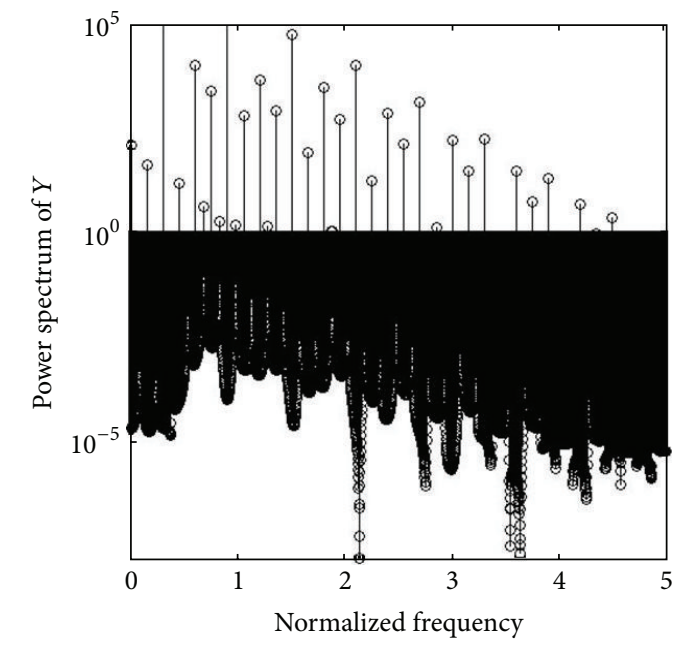

(B)

(e)

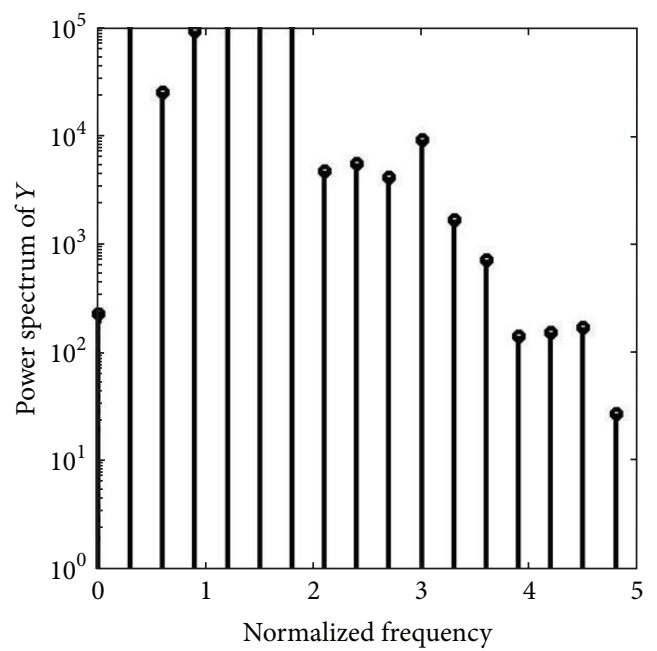

(B)

(f)

Figure 2: Power spectra of coronary artery system at $E=$ (a) 0.1 , (b) 0.3 , (c) 4.3 , (d) 4.5, (e) 5.9, and (f) 16.3 . 


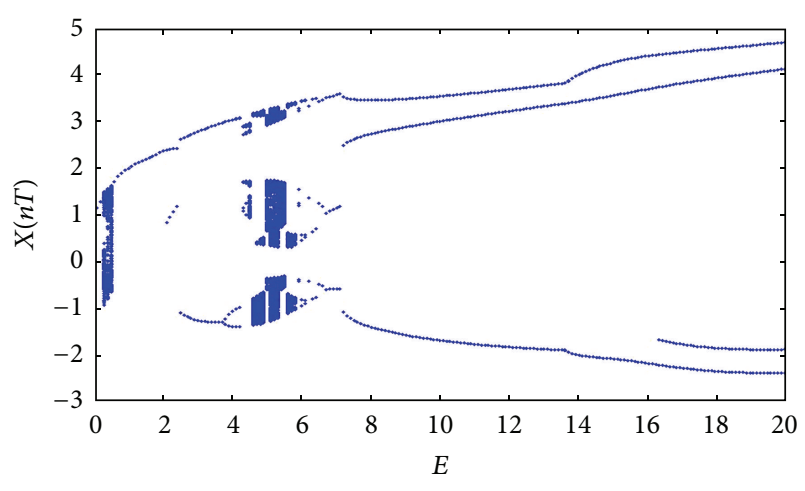

(a)

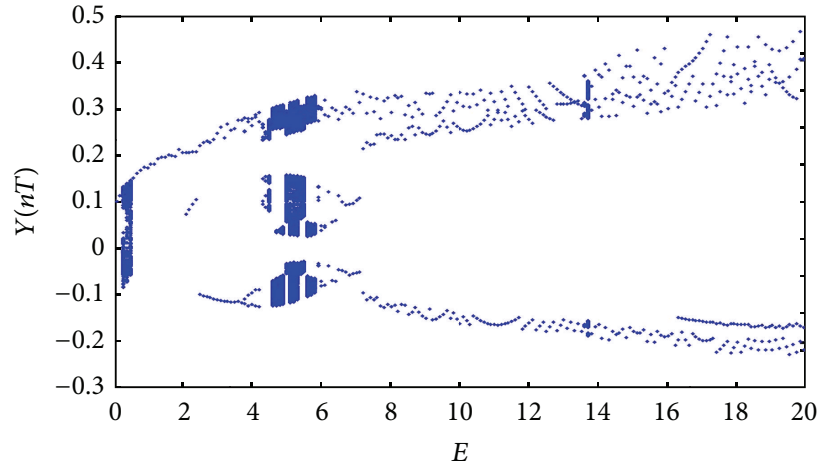

(b)

Figure 3: Bifurcation diagrams for variation of blood vessel diameter $(X)$ and change in blood pressure $(Y)$ using vibrational amplitude $(E)$ as bifurcation parameter: (a) $X(n T)$ and (b) $Y(n T)$.

TABLE 2: Variation of coronary artery system response with vibrational amplitude over interval $0.1 \leqq E \leqq 20.0$.

\begin{tabular}{lc}
\hline$E$ & Dynamic behavior \\
\hline$[0.1,0.3)$ & $\mathrm{T}$ \\
{$[0.3,0.6)$} & Chaos \\
{$[0.6,2.1)$} & $\mathrm{T}$ \\
{$[2.1,3.8)$} & $2 \mathrm{~T}$ \\
{$[3.8,4.3)$} & $4 \mathrm{~T}$ \\
{$[4.3,4.5)$} & $8 \mathrm{~T}$ \\
{$[4.5,5.9)$} & $\mathrm{Chaos}$ \\
{$[5.9,6.0)$} & $8 \mathrm{~T}$ \\
{$[6.0,6.2)$} & $6 \mathrm{~T}$ \\
{$[6.2,16.3)$} & $3 \mathrm{~T}$ \\
{$[16.3,20.0]$} & $4 \mathrm{~T}$ \\
\hline
\end{tabular}

abnormal coronary artery system to a normal coronary artery system.

3.1. Control System Description. Consider the following master-slave coronary artery systems.

Master system is

$$
\begin{gathered}
\dot{x}_{1}=-b x_{1}-c x_{2}, \\
\dot{x}_{2}=-(b+1) \lambda x_{1}-(c+1) \lambda x_{2}+\lambda x_{1}^{3}+E_{1} \cos \omega t
\end{gathered}
$$

and Slave system:

$$
\begin{aligned}
\dot{y}_{1}= & -b y_{1}-c y_{2}, \\
\dot{y}_{2}= & -(b+1) \lambda y_{1}-(c+1) \lambda y_{2} \\
& +\lambda y_{1}^{3}+E_{2} \cos \omega t+\Delta f+u,
\end{aligned}
$$

where $x_{i}$, and $y_{i}(i=1,2)$ are state variables. It is assumed that the master system (4) is a normal coronary artery system and demonstrates chaotic motion in the region of $E_{1} \in[0.3,0.6)$. The slave system (5) is an abnormal coronary artery system and also demonstrates chaotic motion in the region of $E_{2} \epsilon$
$[4.5,5.9) . u(t)$ in (5) is a control input and $\Delta f$ is a bounded unmodelled system structure; that is, $|\Delta f| \leq \alpha$ ( $\alpha$ is positive). Now define the error states as

$$
e_{1}=y_{1}-x_{1} ; \quad e_{2}=y_{2}-x_{2} .
$$

The dynamics of the error system is determined directly from (4)-(5) as follows:

$$
\begin{gathered}
\dot{e}_{1}=-b e_{1}-c e_{2}, \\
\dot{e}_{2}=-(b+1) \lambda e_{1}-(c+1) \lambda e_{2}+\lambda y_{1}^{3} \\
-\lambda x_{1}^{3}+\left(E_{2}-E_{1}\right) \cos (\omega t)+\Delta f+u .
\end{gathered}
$$

From [15], it can be seen that, if (7a) and (7b) are asymptotically stable, then the error states $e_{1}$ and $e_{2}$ will approach zero and the systems (4) and (5) will be synchronized. Therefore, the abnormal chaotic coronary artery system will be controlled to the normal chaotic coronary artery system. The considered goal is that, for any given chaotic coronary artery systems as (4) and (5), a controller is designed such that the resulting tracking error can be driven to zero; that is,

$$
\lim _{t \rightarrow \infty}\left|e_{i}\right| \longrightarrow 0, \quad i=1,2 .
$$

In this study, a fuzzy logic controller (FLC) by Yau and Shieh [15] is used to achieve the control goal. It means that the fuzzy logic control input $u$ (e.g., nitroglycerin) is quickly absorbed; blood vessels dilate and increase blood supply to the heart muscle, which effectively relieves or eliminates angina symptoms.

In consequence, to achieve this control goal for chaotic coronary artery systems with uncertainties, there exist two major phases. First, we let the control input $u(t)=u_{\mathrm{eq}}+u_{L}$ and $u_{\mathrm{eq}}=-\lambda y_{1}^{3}+\lambda x_{1}^{3}+\left(E_{1}-E_{2}\right) \cos (\omega t)$; then the error dynamics becomes

$$
\begin{gathered}
\dot{e}_{1}=-b e_{1}-c e_{2}, \\
\dot{e}_{2}=-(b+1) \lambda e_{1}-(c+1) \lambda e_{2}+\Delta f+u_{L} .
\end{gathered}
$$



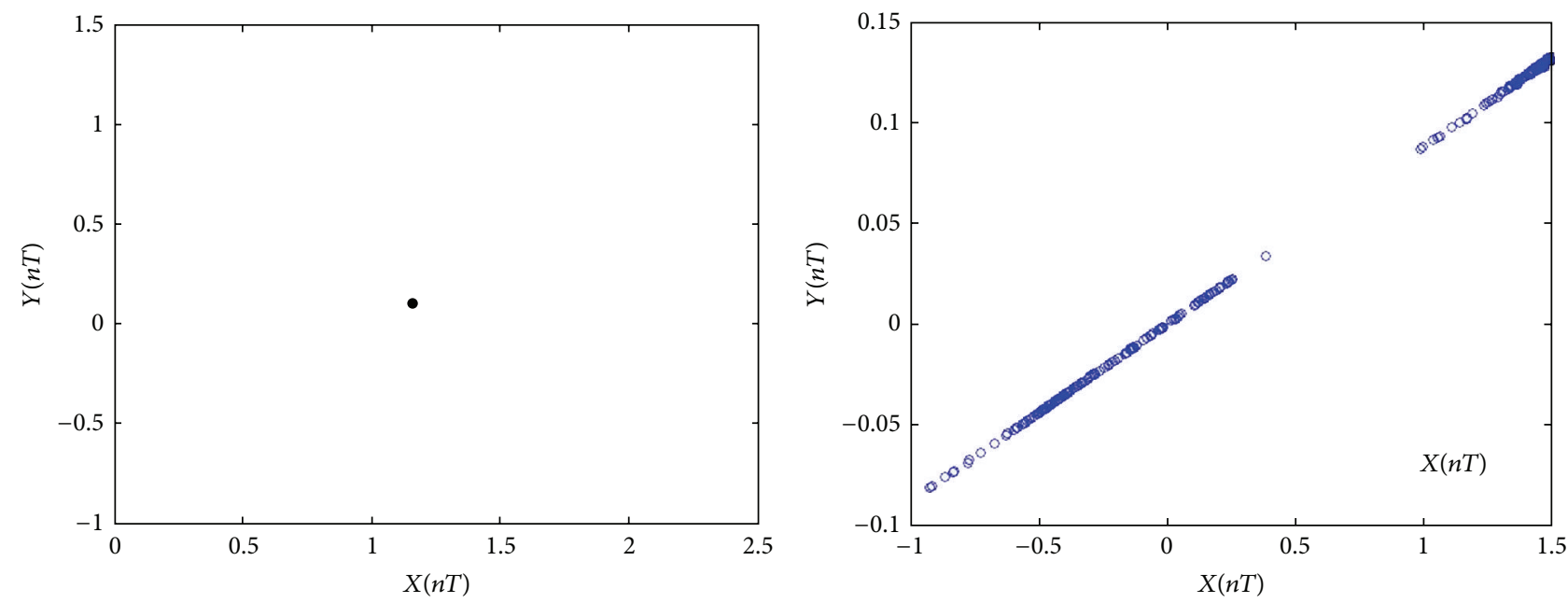

(a)

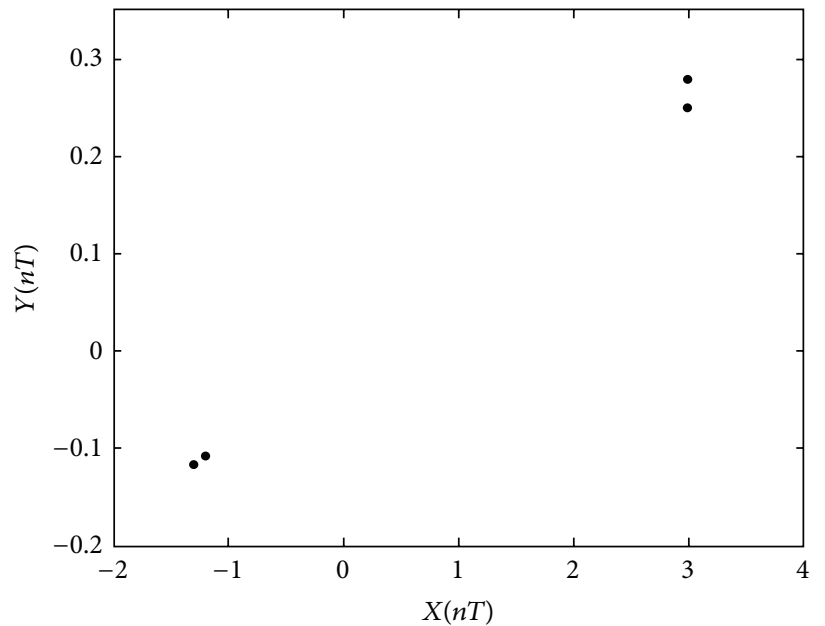

(b)

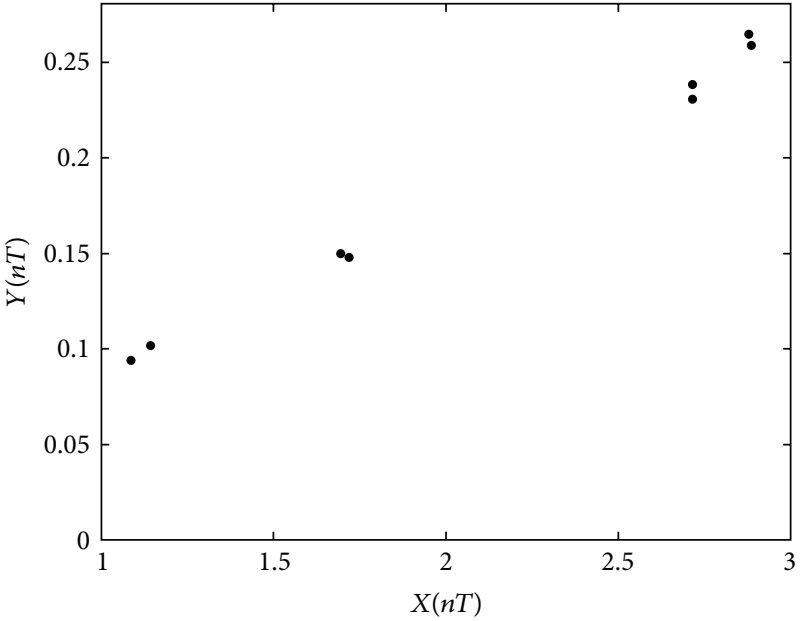

(c)

(d)

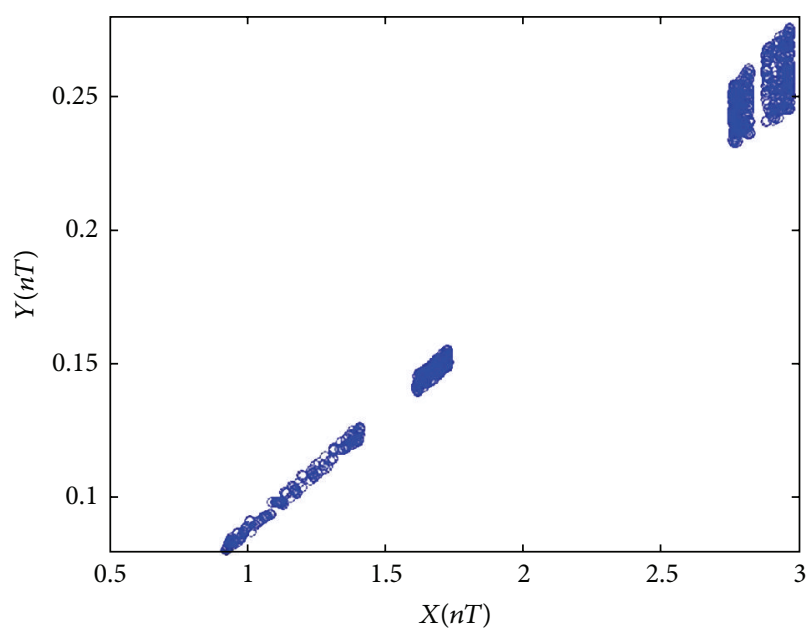

(e)

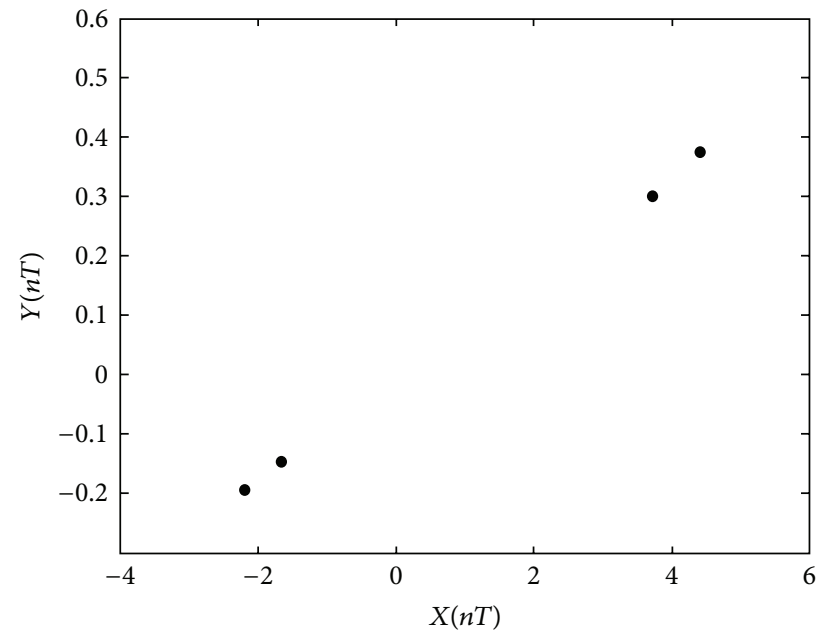

(f)

FIGURE 4: Poincaré maps of coronary artery system at different values of vibrational amplitude (E): (a) 0.1 , (b) 0.3 , (c) 3.8 , (d) 4.3, (e) 4.5, and (f) 16.3 . 


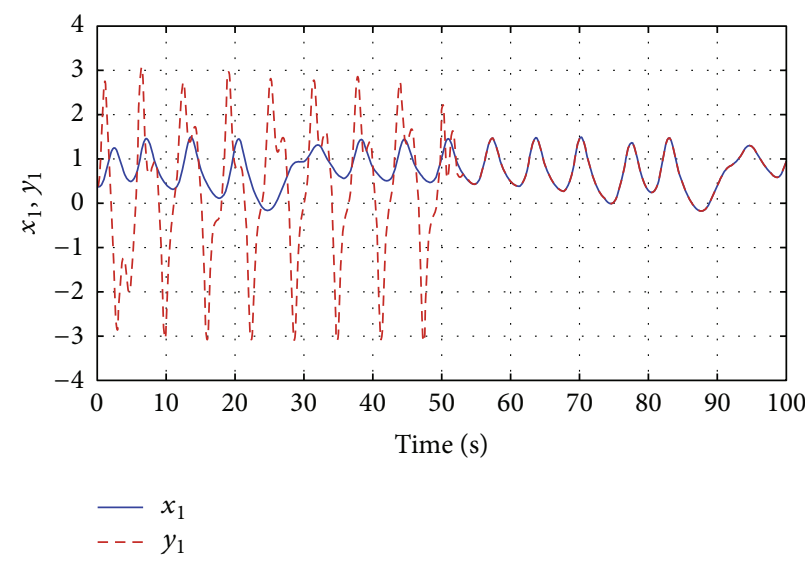

(a)

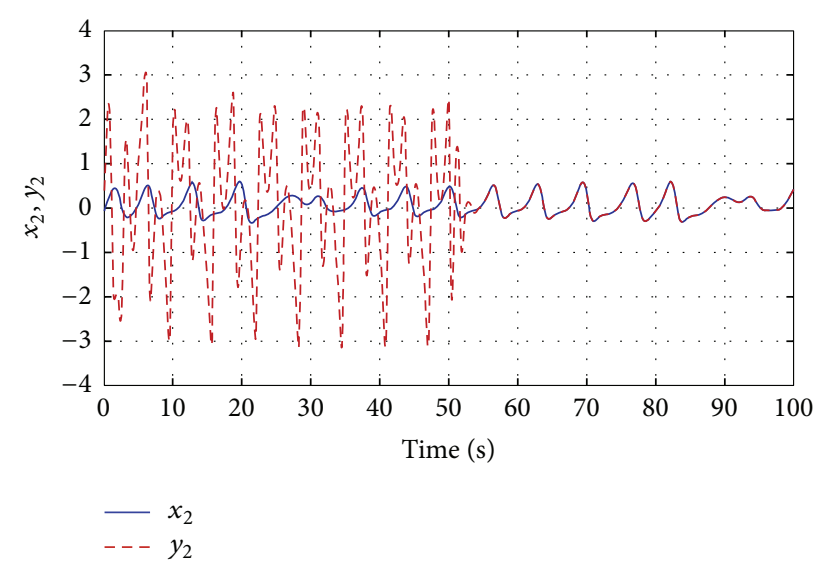

(b)

FIGURE 5: Time responses of chaos synchronization of coronary artery systems: master and slave system outputs are $x_{1}, x_{2}\left(\right.$ solid) and $y_{1}, y_{2}$ (dashed), respectively. The control $u(t)$ is activated at $t=50 \mathrm{sec}$.

According to the state transformation by Yau and Shieh [15], the state transformation is defined as

$$
\left[\begin{array}{l}
\bar{e}_{1} \\
\bar{e}_{2}
\end{array}\right]=\left[\begin{array}{cc}
-\frac{1}{c} & 0 \\
\frac{b}{c} & 1
\end{array}\right]\left[\begin{array}{l}
e_{1} \\
e_{2}
\end{array}\right] .
$$

Substituting (10) into (9a) and (9b) yields

$$
\begin{gathered}
\dot{\bar{e}}_{1}=\bar{e}_{2}, \\
\dot{\bar{e}}_{2}=p_{1} \bar{e}_{1}-p \bar{e}_{2}+\Delta f+u_{L},
\end{gathered}
$$

where $p_{1}=(b-c) \lambda$ and $p=b+(c+1) \lambda$. Second, it needs to determine a FLC such that the error dynamic system (11) is asymptotically stable and the error states $e_{1}$ and $e_{2}$ will approach zero. Therefore, the FLC design process is the same as Duffing-Holmes system studied in [15]. The consequent part in Table 1 is shown in the following:

$$
\begin{gathered}
u_{L 1}=u_{L 4}=u_{L 7}=\left(p_{1}-1\right) \bar{e}_{1}+p \bar{e}_{2}-\alpha, \\
u_{L 3}=u_{L 6}=u_{L 9}=\left(p_{1}-1\right) \bar{e}_{1}+p \bar{e}_{2}+\alpha, \\
u_{L 2}=-\operatorname{sgn}\left(e_{2}\right)+p_{1} \bar{e}_{1}+p \bar{e}_{2}-\alpha, \\
u_{L 8}=-\operatorname{sgn}\left(e_{2}\right)+p_{1} \bar{e}_{1}+p \bar{e}_{2}+\alpha, \\
u_{L 5}=0 .
\end{gathered}
$$

Similarly, we also can show that all the rules in Table 3 also satisfy $\dot{V}<0$ and the proof is omitted. Hence, all of the rules in the FLC can lead to Lyapunov stable subsystems under the same Lyapunov function. Furthermore, the closed-loop rulebased systems (11) are asymptotically stable for each derivate of the Lyapunov function that satisfies $\dot{V}<0$. That is, the error states $e_{1}$ and $e_{2}$ guarantee convergence to zero, and the chaotic coronary artery systems (4) and (5) are synchronized.

3.2. Numerical Results of Synchronization Control. For the overall control systems (4) and (5), the parameters are $b=$
TABLE 3: Rule table of FLC.

\begin{tabular}{lccc}
\hline Rule & & Antecedent & Consequent \\
\hline 1 & $e_{1}$ & $e_{2}$ & $u_{L i}$ \\
2 & $\mathrm{P}$ & $\mathrm{P}$ & $u_{L 1}$ \\
3 & $\mathrm{P}$ & $\mathrm{Z}$ & $u_{L 2}$ \\
4 & $\mathrm{P}$ & $\mathrm{N}$ & $u_{L 3}$ \\
5 & $\mathrm{Z}$ & $\mathrm{P}$ & $u_{L 4}$ \\
6 & $\mathrm{Z}$ & $\mathrm{Z}$ & $u_{L 5}$ \\
7 & $\mathrm{Z}$ & $\mathrm{N}$ & $u_{L 6}$ \\
8 & $\mathrm{~N}$ & $\mathrm{P}$ & $u_{L 7}$ \\
9 & $\mathrm{~N}$ & $\mathrm{Z}$ & $u_{L 8}$ \\
\hline
\end{tabular}

$0.15, c=-1.7, \lambda=-0.65, E_{1}=0.3, E_{2}=4.5$, and $\omega=1$; the master system (4) displaces chaotic behavior. It is supposed that the uncertainty $\Delta f=0.2 \cdot \cos \left(\pi y_{1}\right)$; that is $|\Delta f| \leq \alpha=0.2$. The simulation results with initial conditions $x_{1}(0)=0.4, x_{2}(0)=-0.1, y_{1}(0)=0.2$, and $y_{2}(0)=$ 0.2 are shown in Figures $5-7$. Figure 5 shows that the slave system and the master system can reach synchronization with control operation. In addition, the time responses of error states and control input are shown in Figures 6 and 7. It can be seen that the system error states are regulated to zero asymptotically even when the overall system is undergoing system uncertainty.

\section{Conclusion}

This study has applied the differential transformation method to investigate the dynamic behavior of a CA system. Phase trajectories, Poincaré maps, and bifurcation diagrams have been used to characterize the dynamic response of the system as a function of the vibrational amplitude and to detect the onset of chaotic motion. In general, the results have shown that, as the vibrational amplitude is increased from 0.1 to 


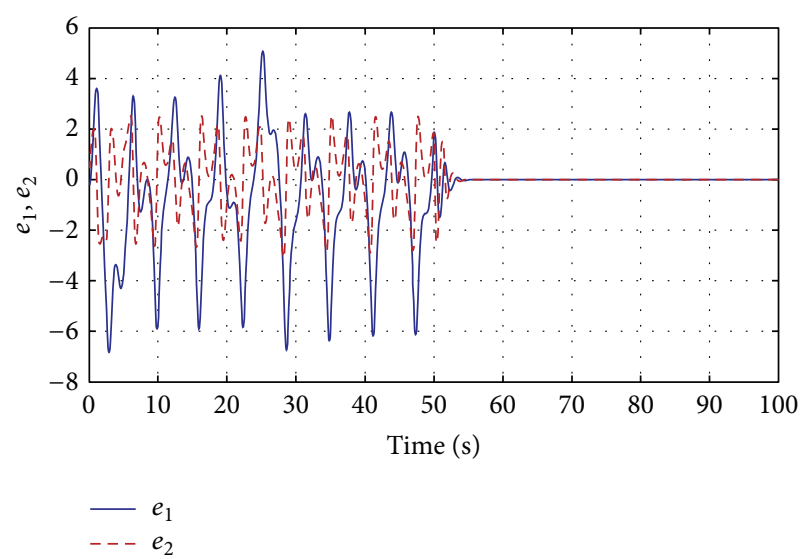

FIGURE 6: The time response of error states with control $u(t)$ activated at $t=50 \mathrm{sec}$.

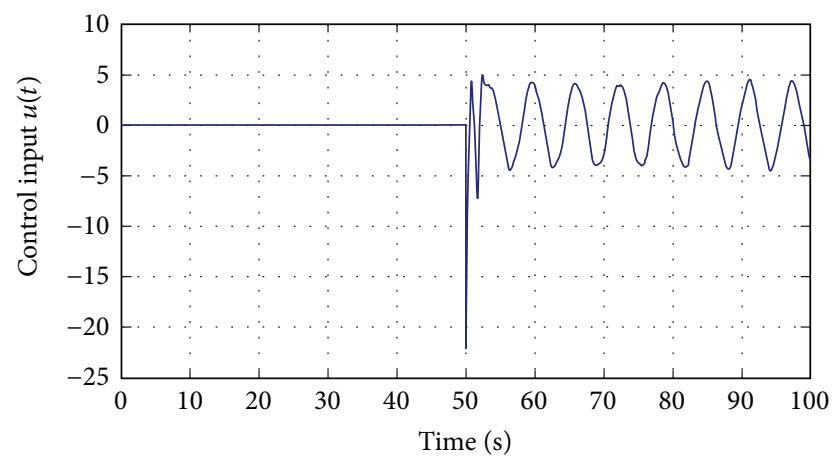

FIGURE 7: The control action versus time with control $u(t)$ activated at $t=50 \mathrm{sec}$.

20.0, the system motion changes initially from $T$-periodic to chaotic, $T$-periodic, $2 T$-periodic, and multiperiodic and then from $8 T$-periodic to chaos and is finally transferred to multiperiodic motion with windows of periodic motion.

In this paper, nonlinear FLC theory has been exploited to design a controller for chaos synchronization with system uncertainties. It can synchronize the abnormal CA system to a normal CA system. It shows that the FLC in this paper is realizable for implementation and it can reduce the actuator saturation phenomenon in real physics system. The other types of CA synchronization control could also be synchronized by using the same control scheme proposed in this study.

\section{Appendix}

Let $x(t)$ be analytic in the time domain $T$. Further, let:

$$
\phi(t, k)=\frac{d^{k} x(t)}{d t^{k}}, \quad \forall t \in T .
$$

At $t=t_{i}, \phi(t, k)=\phi\left(t_{i}, k\right)$, where $k$ belongs to a set of nonnegative integers, denoted by the $K$ domain. Therefore, (A.1) can be rewritten as

$$
X_{i}(k)=\phi\left(t_{i}, k\right)=\left[\frac{d^{k} x(t)}{d t^{k}}\right]_{t=t_{i}}, \quad \forall k \in K,
$$

where $X(k)$ represents the spectrum of $x(t)$ at $t=t_{i}$ in the $K$ domain.

If $x(t)$ is analytic, then $x(t)$ can be represented as

$$
x(t)=\sum_{k=0}^{\infty} \frac{\left(t-t_{i}\right)^{k}}{k !} X(k) .
$$

Note that this equation represents the inverse transformation of $X(k)$.

If $X(k)$ is defined as

$$
X(k)=M(k)\left[\frac{d^{k} q(t) x(t)}{d t^{k}}\right]_{t=t_{0}},
$$

where $k=0,1,2, \ldots, \infty$,

then the function $x(t)$ can be described as

$$
x(t)=\frac{1}{q(t)} \sum_{k=0}^{\infty} \frac{\left(t-t_{i}\right)^{k}}{k !} \frac{X(k)}{M(k)},
$$

where $M(k) \neq 0$ and $q(t) \neq 0 . M(k)$ is the weighting factor and $q(t)$ is regarded as a kernel corresponding to $x(t)$. If $M(k)=1$ and $q(t)=1$, then (A.2), (A.4), (A.3), and (A.5) are equivalent. In this paper, transformation is applied with $M(k)=\widetilde{H}^{k} / k !$ and $q(t)=1$, where $\widetilde{H}$ is the time horizon of interest. $X(k)$ is given by

$$
X(k)=\frac{\widetilde{H}^{k}}{k !}\left[\frac{d^{k} x(t)}{d t^{k}}\right]_{t=t_{0}}, \quad \text { where } k=0,1,2, \ldots, \infty .
$$

Using the differential transformation method, a differential equation in the domain of interest can be transformed to an algebraic equation in the $K$ domain and $x(t)$ can be obtained by the finite-term Taylor series plus a remainder; that is,

$$
\begin{aligned}
x(t) & =\frac{1}{q(t)} \sum_{k=0}^{n} \frac{\left(t-t_{0}\right)^{k}}{k !} \frac{X(k)}{M(k)}+R_{n+1} \\
& =\sum_{k=0}^{n}\left(\frac{t-t_{0}}{\widetilde{H}}\right)^{k} X(k)+R_{n+1} .
\end{aligned}
$$

In order to accelerate the rate of convergence and improve the accuracy of the calculations, the overall $t$ domain can be split into a number of subdomains. The differential equation can then be solved in each domain.

The approach described above is used to split the time domain into a total of $n$ subdomains, as shown in Figure 8. Considering function $x(t)$ in the first subdomain $(0 \leq t \leq$ $\left.t_{1}, t_{0}=0\right)$, the one-dimensional differential transformation is given by

$$
x(t)=\sum_{k=0}^{n}\left(\frac{t-t_{0}}{H_{0}}\right)^{k} X_{0}(k)
$$

where $X_{0}(0)=x_{0}$. The differential transformation and system dynamic equations can be solved for the first subdomain 


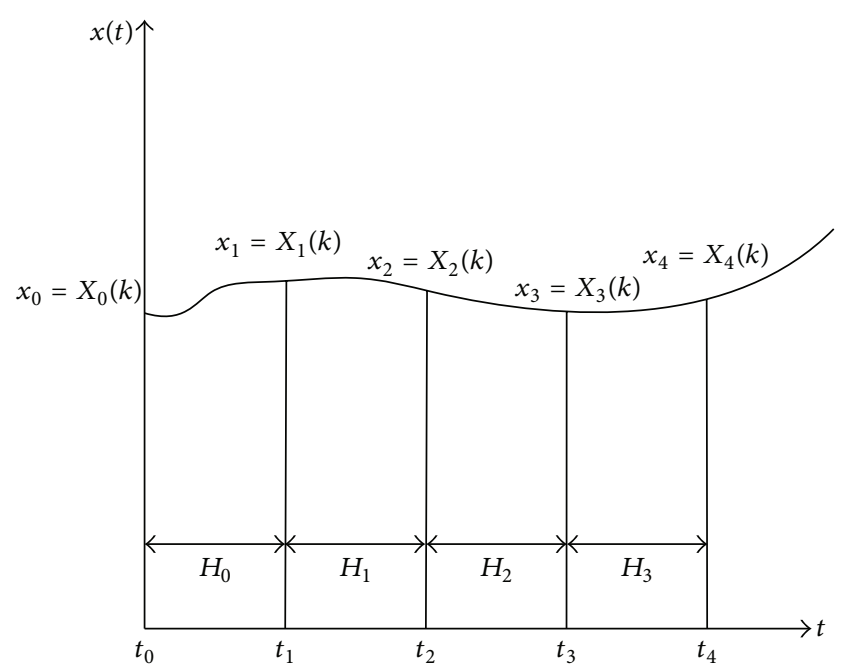

FIgURE 8: Time step diagram.

and $X_{0}(k)$ can be solved entirely in the first subdomain. The end point of function $x(t)$ in the first subdomain is $x_{1}$, and the value of $t$ is $H_{0}$. Therefore, $x_{1}(t)$ is obtained by the differential transformation method as

$$
x_{1}=x\left(H_{0}\right)=\sum_{k=0}^{\infty} X_{0}(k)
$$

Note that $x_{1}$ represents the initial condition in the second subdomain and therefore $X_{1}(0)=x_{1}$. The function $x(t)$ can be expressed in the second subdomain as

$$
x_{2}=x\left(H_{1}\right)=\sum_{k=0}^{\infty} X_{1}(k)
$$

In general, the function $x(t)$ can be expressed in the (I-1) subdomain as

$$
\begin{aligned}
x_{i} & =x_{i-1}+\sum_{k=1}^{\infty} X_{i-1}(k) \\
& =X_{i-1}(0)+\sum_{k=1}^{\infty} X_{i-1}(k)=1,2,3, \ldots, n .
\end{aligned}
$$

Using the $T$ spectra method described above, function $x(t)$ can be solved throughout the entire domain.

Table 4 shows differential transformation. The symbol “» denotes the differential operator, and “ $\otimes$ " denotes the convolution operation in the $K$ domain.

If $f(t)$ and $g(t)$ are two uncorrelated functions of $t$, and $F(k)$ and $G(k)$ are the corresponding transformation functions, respectively, then the basic properties of the differential transformation are as follows.

(1) Linearity. If $F(k)=D[f(t)], G(k)=D[g(t)]$, and $C_{1}$ and $C_{2}$ are independent of $t$ and $k$, then

$$
D\left[C_{1} f(t)+C_{2} g(t)\right]=C_{1} F(k)+C_{2} G(k) .
$$

TABLE 4: Operation in the $K$ domain with $M(k)=\widetilde{H}^{k} / k !, q(t)=1$.

\begin{tabular}{ll}
\hline Operator & $X(k)=\frac{\widetilde{H}^{k}}{k !}\left(\frac{\partial^{k} x(t)}{\partial t^{k}}\right)_{t=0}$ \\
Spectrum $X(k)$ & $x(t)=\sum_{l=0}^{k}\left[X(k)\left(\frac{t}{\widetilde{H}}\right)^{k}\right]$ \\
Function $x(t)$ & $X(k) \otimes Y(k)=\sum_{l=0}^{k} X(l) Y(k-l)$ \\
Derivative & $\wedge(k)=\frac{k+1}{\widetilde{H}} X(k+1)$
\end{tabular}

(2) Convolution. If $z(t)=f(t) g(t), f(t)=D^{-1}[F(k)]$, and $g(t)=D^{-1}[G(k)]$, then

$$
\begin{aligned}
D[z(t)] & =D[f(t) g(t)]=F(k) \otimes G(k) \\
& =\sum_{l=0}^{k} F(l) G(k-l)=\sum_{l=0}^{k} F(k-l) G(l) .
\end{aligned}
$$

Therefore, the differential transform of $f^{m}(t)$, where $m$ is a positive integer, can be obtained as

$$
\begin{aligned}
D\left[f^{m}(t)\right] & =F^{m}(k)=F^{m-1}(k) \otimes F(k) \\
& =\sum_{l=0}^{k} F^{m-1}(l) F(k-l) .
\end{aligned}
$$

(3) Derivative. If $f(t)$ and its derivatives $f^{\prime}(t), f^{\prime \prime}(t), \ldots$, $f^{(n)}(t)$ are continuous functions for the interval $[0, \widetilde{H}]$, then

$$
D\left[\frac{d^{n} f(t)}{d t^{n}}\right]=\frac{(k+1)(k+2), \ldots,(k+n)}{\widetilde{H}^{n}} F(k+n) .
$$

\section{Conflict of Interests}

The authors declare no conflict of interests.

\section{Acknowledgment}

The financial support provided to this study by the National Science Council of Taiwan under Grant no. NSC100-2628-E269-016-MY2 is greatly appreciated.

\section{References}

[1] S. M. Jadhav, S. L. Nalbalwar, and A. A. Ghatol, "ECG arrhythmia classification using modular neural network model," in Proceedings of the IEEE EMBS Conference on Biomedical Engineering and Sciences (IECBES '10), pp. 62-66, December 2010.

[2] D. A. Coast, R. M. Stern, G. G. Cano, and S. A. Briller, "An approach to cardiac arrhythmia analysis using hidden Markov models," IEEE Transactions on Biomedical Engineering, vol. 37, no. 9, pp. 826-836, 1990. 
[3] C. Blondel, G. Malandain, R. Vaillant, and N. Ayache, "Reconstruction of coronary arteries from a single rotational X-ray projection sequence," IEEE Transactions on Medical Imaging, vol. 25, no. 5, pp. 653-663, 2006.

[4] D. Gao, M. Madden, D. Chambers, and G. Lyons, "Bayesian ANN classifier for ECG arrhythmia diagnostic system: A Comparison Study," in Proceedings of the International Joint Conference on Neural Networks (IJCNN '05), pp. 2383-2388, August 2005.

[5] I. Khalil and F. Sufi, "CardioGrid: ECG analysis on demand to detect cardiovascular abnormalities," in Proceedings of the 9th International Conference on Information Technology and Applications in Biomedicine (ITAB '09), November 2009.

[6] Z. Sun and W. Ding, "Analysis of coronary arterial dynamics from X-ray angiographic sequences," in Proceedings of the International Symposium on Computational Intelligence and Design (ISCID '09), pp. 201-204, December 2009.

[7] J. Puentes, C. Roux, M. Garreau, and J. L. Coatrieux, "Dynamic feature extraction of coronary artery motion using DSA image sequences," IEEE Transactions on Medical Imaging, vol. 17, no. 6, pp. 857-871, 1998.

[8] X. P. Guan, Z. P. Fan, and C. L. Chen, Chaotic Control and Its Application in Secret Communication, National Defense Industry Press, Beijing, China, 2002.

[9] C. Y. Gong, Y. M. Li, and X. H. Sun, "Backstepping control of synchronization for biomathematical model of muscular blood vessel," Journal of Applied Sciences, vol. 24, pp. 604-607, 2006.

[10] C.-C. Wang, M.-J. Jang, and Y.-L. Yeh, "Bifurcation and nonlinear dynamic analysis of a flexible rotor supported by relative short gas journal bearings," Chaos, Solitons and Fractals, vol. 32, no. 2, pp. 566-582, 2007.

[11] C.-C. Wang, "Theoretical and nonlinear behavior analysis of a flexible rotor supported by a relative short herringbone-grooved gas journal-bearing system," Physica D, vol. 237, no. 18, pp. 2282-2295, 2008.

[12] C.-C. Wang, "Application of a hybrid method to the nonlinear dynamic analysis of a flexible rotor supported by a spherical gaslubricated bearing system," Nonlinear Analysis: Theory, Methods \& Applications, vol. 70, no. 5, pp. 2035-2053, 2009.

[13] C. Bianca and L. Rondoni, "The nonequilibrium Ehrenfest gas: a chaotic model with flat obstacles?" Chaos, vol. 19, no. 1, Article ID 013121, 2009.

[14] O. G. Jepps, C. Bianca, and L. Rondoni, "Onset of diffusive behavior in confined transport systems," Chaos, vol. 18, no. 1, Article ID 013127, 2008.

[15] H.-T. Yau and C.-S. Shieh, "Chaos synchronization using fuzzy logic controller," Nonlinear Analysis: Real World Applications, vol. 9, no. 4, pp. 1800-1810, 2008. 


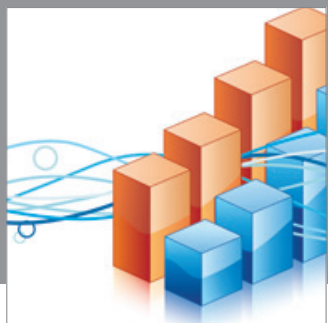

Advances in

Operations Research

mansans

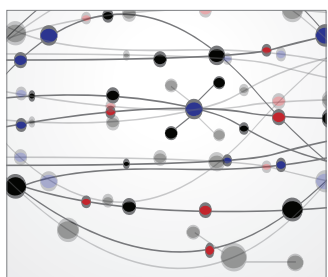

The Scientific World Journal
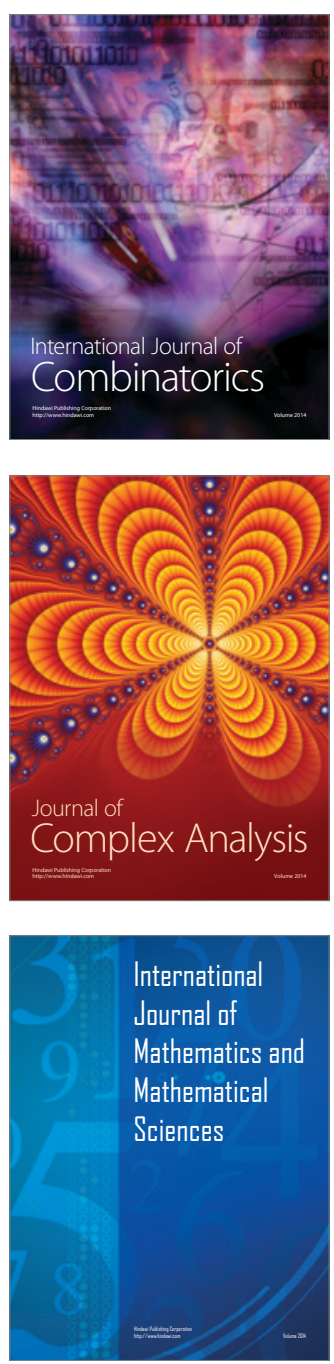
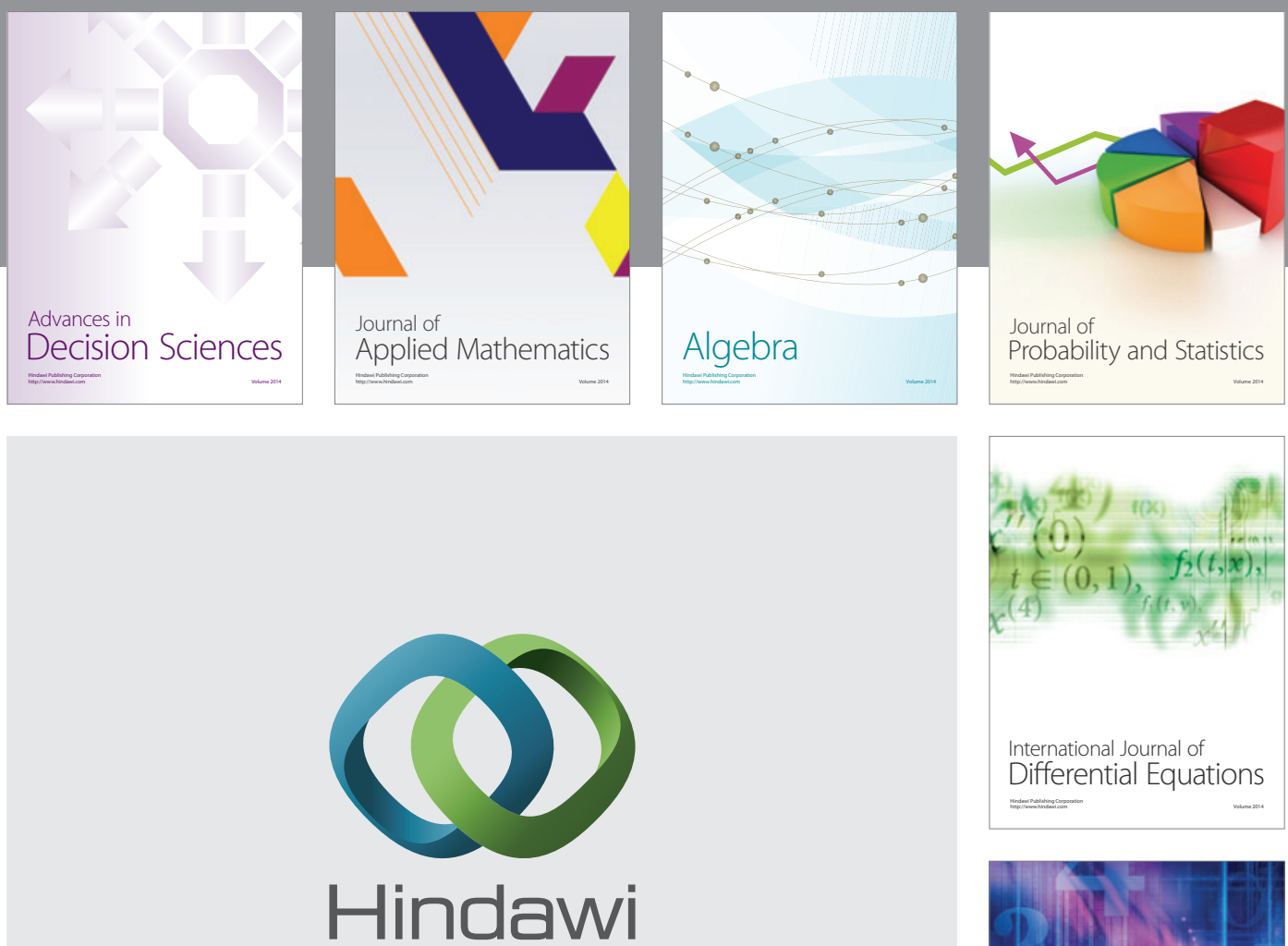

Submit your manuscripts at http://www.hindawi.com
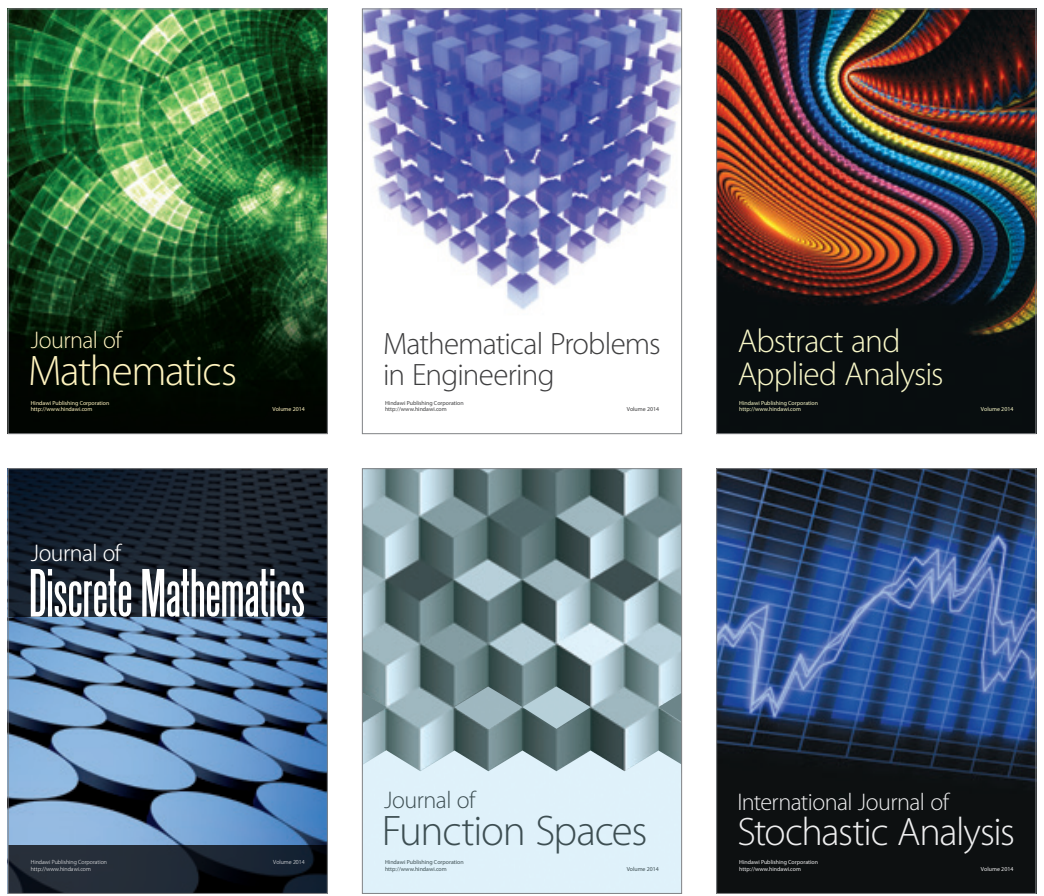

Journal of

Function Spaces

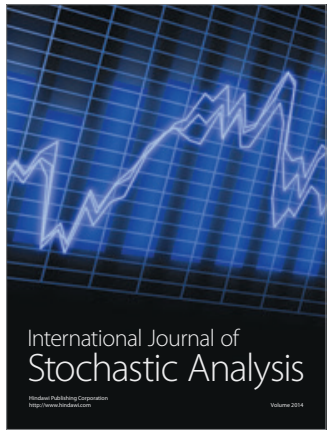

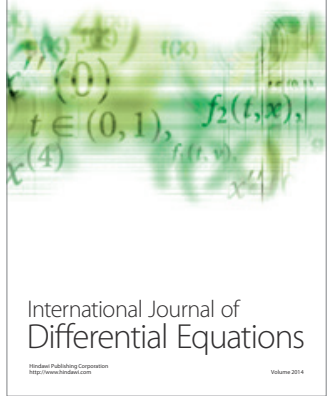
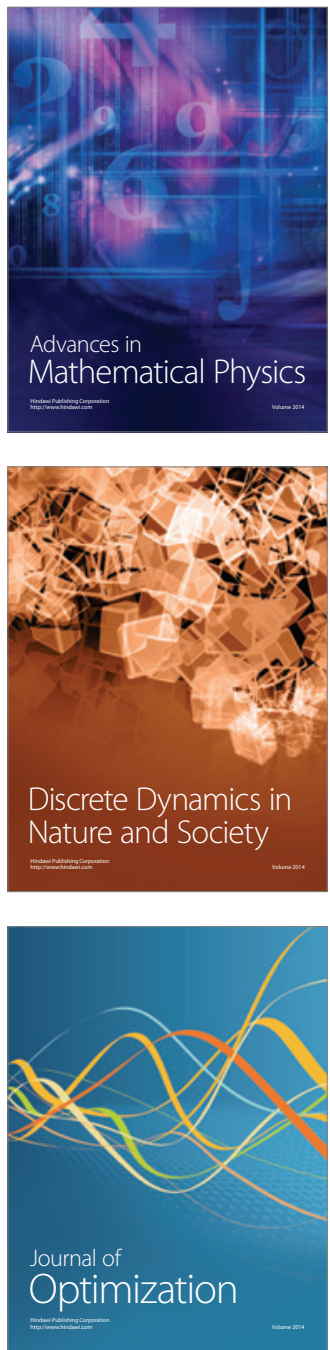